\title{
Das Grundrecht der Meinungsfreiheit im Lichte des „Historikerstreits“
}

Mit Beschluss vom 4. November 2009 hat das Bundesverfassungsgericht den Straftatbestand der Störung des ,öffentlichen Frieden[s] in einer die Würde der Opfer verletzenden Weise“ durch Billigung, Verherrlichung oder Rechtfertigung der ,nationalsozialistische[n] Gewalt- und Willkürherrschaft" (§ 130 Abs. 4 StGB) für verfassungsgemäß erklärt. In den amtlichen Leitsätzen wird dazu zunächst festgestellt, es handele sich bei der Strafbestimmung zwar nicht um ein allgemeines Gesetz, für das Art. 5 Abs. 2 GG eine Einschränkung der Meinungsfreiheit zulasse. Das sei aber, wie der erste Leitsatz weiter ausführt, im vorliegenden Falle unschädlich: „Angesichts des sich allgemeinen Kategorien entziehenden Unrechts und des Schreckens, die die nationalsozialistische Herrschaft über Europa und weite Teile der Welt gebracht hat, und der als Gegenentwurf hierzu verstandenen Entstehung der Bundesrepublik Deutschland ist Art. 5 Abs. 1 und 2 GG für Bestimmungen, die der propagandistischen Gutheißung der nationalsozialistischen Gewalt- und Willkürherrschaft Grenzen setzen, eine Ausnahme vom Verbot des Sonderrechts für meinungsbezogene Gesetze immanent." In vorweggenommener Reaktion auf die zu erwartenden Einwände gegen diese Feststellung heißt es im zweiten Leitsatz weiter: „Die Offenheit des Art. 5 Abs. 1 und 2 GG für derartige Sonderbestimmungen nimmt den materiellen Gehalt der Meinungsfreiheit nicht zurück. Das Grundgesetz rechtfertigt kein allgemeines Verbot der Verbreitung rechtsradikalen oder auch nationalsozialistischen Gedankenguts schon in Bezug auf die geistige Wirkung seines Inhalts. " ${ }^{1}$ Gleichwohl hat die Literatur überwiegend kritisch bis ablehnend auf den bundesverfassungsgerichtlichen Beschluss reagiert. Die Entscheidung bewege sich an den „Grenzen der Dogmatik“2 und sei zumindest mit „,methodischen Zweifeln" ${ }^{\text {"3 }}$ behaftet. „Von einem Verständnis der Meinungsfreiheit als wirklicher Geistesfreiheit“, von dem das Gericht dem eigenen Bekunden nach ausgehe, bleibe „wenn es hart auf hart kommt, nicht viel zurück“. ${ }^{4}$ Die in der „maßgeblich vom Ergebnis her gedachten "5 Entscheidung entwickelte „Sonderdogmatik gerade und ausschließlich für [...] rechtsradikale Verfassungsfeinde“ bleibe „dogmatisch unbefriedigend“. 6 Die Rechtsprechung zur Meinungsfreiheit gerate ,dadurch in eine rechtsstaatlich bedenkliche Schieflage". 7

1 BVerfGE 124, 300; Zur ,,verhalten wohlwollend[en]“ Aufnahme der Entscheidung in der Tagespresse S. Martini, JöR, n.F. 51 (2011), 279 (281).

2 O. Lepsius, Jura 2010, 527 (532); an diesen anschließend M. Hong, DVB1. 2010, 1267 (1271, 1276) m.w.N.; zurückhaltender R. Zimmermann, NJ 2011, 145 (153).

3 C. Degenhart, JZ 2010, 306 (310); vgl. auch W. Höfling/S. Augsberg, JZ 2010, 1088 (1095): „argumentative Inkonsistenz“; noch weitergehend T. Barczak, StudZR 2010, 309 (319): „rechtsdogmatisches Chaos“.

4 U. Volkmann, NJW 2010, 417 (429).

5 T. Barczak, StudZR 2010, 309; ebenso A. Kirsch, NWVB1. 2010, 136 (139): „nur [...] ergebnisorientiert".

6 L. Michael, ZJS 2010, 155 (164), unter ergänzender Bezugnahme auf Art. 3 Abs. 3 GG.

7 J. P. Schaefer, DÖV 2010, 379. 
Konkret entzündet sich die Kritik insbesondere an der argumentativen Heranziehung der Singularität der nationalsozialistischen Gewalt- und Willkürherrschaft, mit der das Bundesverfassungsgericht die Ausnahme vom Sonderrechtsverbot im Rahmen des Art. 5 Abs. 2 GG letztlich begründet. Der ungeschriebene Ausnahmetatbestand bilde „in der Entscheidung einen erratischen Block, kaum in gewohnten grundrechtsdogmatischen Kategorien fassbar,“ und sei zwar zunächst „,erkennbar nicht als auf andere Fälle übertragbar", sondern ,abschließend gedacht“, also „weder einer Ausweitung noch einer Übertragung auf andere Konstellationen zugänglich“. ${ }^{8}$ Gleichwohl besitze die „Einzelfall-Bereichsausnahme“" durchaus das Potenzial, sich zukünftig zu einem neuen „Muster verfassungskonformen Meinungssonderrechts“ fortzuentwickeln. ${ }^{9}$

Der Verweis des Bundesverfassungsgerichts auf die Singularität der nationalsozialistischen Verbrechensherrschaft weckt Erinnerungen an den „Historikerstreit“, der vor gut 20 Jahren genau um diese Frage geführt wurde. ${ }^{10}$ Diese publizistische „Großkontroverse“ wird zwar im Rückblick als „,konstitutiver Bestandteil des historischen Bewusstseins der Bundesrepublik Deutschland" bezeichnet. ${ }^{11}$ Dennoch sieht man die dort „hin und hergewendete“ - und letztlich auf überwiegende Zustimmung gestoßene ${ }^{12}$ „These von der Singularität der Naziverbrechen“ im vorliegenden verfassungsrechtlichen Kontext als irrelevant an, denn sie rechtfertige heute keinesfalls „,den daraus abgeleiteten juristischen Schluss, Konflikte um rechtsradikale Propaganda seien mit den herkömmlichen, für die Meinungsfreiheit entwickelten Regeln nicht zu erfassen". ${ }^{13}$ Dass das Bundesverfassungsgericht dies nun anders zu sehen scheint, wirft aus juristischer Sicht vor allem zwei Fragen auf: Neben der in der Literatur vorrangig problematisierten dogmatischen Konstruktion sind insbesondere die möglichen Auswirkungen auf andere Fallkonstellationen klärungsbedürftig.

8 M. Hong, ZaöRV 70 (2010), 73 (118 [mit Auflistung der singularitätsbetonenden Entscheidungspassagen], $120 \mathrm{f} ., 124 \mathrm{f}$.); ebenso ders., DVBl. 2010, 1267 (1271 f., 1276).

9 J. P. Schaefer, DÖV 2010, 379 (385); andeutungsweise auch M. Hong, ZaöRV 70 (2010), 73 (125); ders., DVBl. 2010, 1267 (1271).

10 Im Sammelband von R. Augstein u.a., „Historikerstreit“, 1987, findet sich eine umfassende zeitgenössische „Dokumentation der Kontroverse um die Einzigartigkeit der nationalsozialistischen Judenvernichtung" (so der Untertitel); vgl. bes. E. Nolte, ebd., 13 (32 f.), mit der ursprünglichen These aus dem Jahre 1980, wonach die nationalsozialistische Judenvernichtung ,irrationaler“, „,entsetzlicher“ und ,abstoßender“ als die „Vernichtungsvorgänge der Russischen Revolution“ gewesen sei, doch ,all das begründet zwar Singularität, ändert aber nichts an der Tatsache, daß die sogenannte Judenvernichtung des Dritten Reiches eine Reaktion oder verzerrte Kopie und nicht ein erster Akt oder das Original war", dazu die Kritik von J. Habermas, ebd., 62 (71): „Die Nazi-Verbrechen verlieren ihre Singularität dadurch, daß sie als Antwort auf [...] bolschewistische Vernichtungsdrohungen mindestens verständlich gemacht werden. Auschwitz schrumpft auf das Format einer technischen Innovation“"

11 V. Kronenberg, in: ders. (Hrsg.), Zeitgeschichte, Wissenschaft und Politik, 2008, 7 f. m.w.N.

12 Plakativ E. Jesse, Cicero 10/2006, 62: „letzter großer Diskurstriumph“ der ,intellektuellen Linken".

13 H. Meier, Merkur 2010, 539 (541). 


\section{Dogmatische Rekonstruktion des Art. 5 Abs. 2 GG}

Nach der herkömmlichen Formel zu Art. 5 Abs. 2 GG aus dem Lüth-Urteil in Übernahme der schon zur Weimarer Zeit vertretenen Auffassung sind unter allgemeinen Gesetzen ,alle Gesetze zu verstehen [...], die, nicht eine Meinung als solche verbieten, die sich nicht gegen die Äußerung der Meinung als solche richten', die vielmehr, dem Schutze eines schlechthin, ohne Rücksicht auf eine bestimmte Meinung, zu schützenden Rechtsguts dienen', dem Schutze eines Gemeinschaftswerts, der gegenüber der Betätigung der Meinungsfreiheit den Vorrang hat". ${ }^{14}$ Diese Formel enthält in ihrem ersten Teil die sog. Sonderrechtslehre mit dem Gebot der Meinungsneutralität gesetzlicher Beschränkungen der Meinungsfreiheit und in ihrem zweiten Teil (bestehend aus dem letzten Nebensatz) die sog. Abwägungslehre, die dem im Rahmen der grundrechtlichen Schrankendogmatik allgemein anerkannten Verhältnismäßigkeitsgrundsatz entspricht. ${ }^{15}$ Das Bundesverfassungsgericht beschäftigt sich in seinem aktuellen Beschluss zunächst mit der Sonderrechtslehre und hält an der bisherigen Definition als solcher fest. Die darin enthaltene Anforderung an allgemeine Gesetze ist in doppelter Weise, erst negativ (,nicht ..."), dann positiv (,,vielmehr ..."), formuliert. In einer wichtigen Präzisierung der bisherigen Rechtsprechung stellt das Bundesverfassungsgericht dazu fest, dass es keineswegs ausreicht, positiv festzustellen, dass „eine Norm ein anerkanntes Rechtsgut schützt". ${ }^{16}$ Dieser Feststellung, die sich zugleich bereits auf den legitimen Eingriffszweck als Teil der Verhältnismäßigkeitsprüfung im Rahmen der Abwägungslehre bezieht, ${ }^{17}$ kann als solcher allenfalls eine Indizwirkung für die Annahme eines meinungsneutralen Gesetzes zukommen. Zwar bleibt das Vorliegen eines anderen, meinungsunabhängigen Schutzguts schon deshalb immer die notwendige Kehrseite der Meinungsneutralität, weil ein Gesetz niemals Selbstzweck sein kann. ${ }^{18}$ Entscheidend ist aber letztlich, dass die (schutzgut- oder äußerungsbezogene) ,Fassung der Norm [...] allein an dem zu schützenden Rechtsgut ausgerichtet sein darf, nicht aber an einem Wertoder Unwerturteil hinsichtlich der konkreten Haltungen oder Gesinnungen". ${ }^{19}$ Es reicht also nicht aus, dass ein gegen bestimmte Meinungen gerichtetes Gesetz zugleich ein meinungsunabhängiges Gut schützt, sondern dies muss ausschließlicher Gesetzeszweck sein. Eine Beschränkung des Beleidigungstatbestandes ( 185 StGB), der immer zumindest auch dem Schutz der persönlichen Ehre dient, auf den Schutz von Sympathi-

14 BVerfGE 7, 198 (209f.).

15 Zusammenfassend etwa B. Pieroth/B. Schlink, Grundrechte, 26. Aufl., 2010, Rn. 637 ff.; grundsätzliche Kritik an der Bekräftigung der Verbindung von Sonderrechts- und Abwägungslehre im Wunsiedel-Beschluss - aus rechtsvergleichender Perspektive - bei K.-H. Ladeur, K \& R 2010, 642 (643 ff.).

16 BVerfGE 124, 300 (321 ff.).

17 BVerfGE 124, 300 (331 ff.).

18 Allgemeiner - in Bezug auf den Staat - BVerfGE 123, 267 (346).

19 BVerfGE 124, 300 (324 [Hervorh. nicht i. Orig.]); vgl. auch M. Hong, DVBl. 2010, 1267 ff.: „Verbot der Standpunktdiskriminierung“; S. Martini, JöR, n.F. 59 (2011), 279 (284 f.): ,doppelte Blindheitsprüfung“". 
santen der aktuellen Regierungsmehrheit wäre danach nicht nur im Hinblick auf Art. 3 GG unzulässig. ${ }^{20}$

Diesem ausdifferenzierten Maßstab genügt die Bestimmung des $\S 130$ Abs. 4 StGB nicht in vollem Umfang. Denn sie schützt zwar auch ein meinungsunabhängiges Gut, nämlich den öffentlichen Frieden. ${ }^{21}$ Aber dies geschieht nicht in absolut meinungsneutraler Weise, da die Bestimmung zugleich und primär gegen eine bestimmte Meinung, nämlich die ,propagandistische Gutheißung der nationalsozialistischen Gewalt- und Willkürherrschaft", gerichtet ist. ${ }^{22}$ Die Ausnahme vom Gebot der Meinungsneutralität nach Art. 5 Abs. 2 GG, die das Bundesverfassungsgericht speziell und nur für diesen Fall postuliert, ist allerdings ihrerseits keine absolute, sondern sie gilt nur für die negative Komponente (,nicht gegen eine bestimmte Meinung gerichtet"). Die positive Anforderung (,dem Schutze eines schlechthin [d.h. meinungsunabhängig] zu schützenden Rechtsguts dienend“) wird hingegen aufrechterhalten. Der Schutz des meinungsunabhängigen Rechtsgutes wird hier also ausnahmsweise nicht als alleiniger Normzweck verlangt, aber in zulässiger Weise anti-nationalsozialistisch formulierte Normen müssen immerhin - wie dies $\S 130$ Abs. 4 StGB tut - zugleich einen solchen Schutz bezwecken und in diesem Rahmen den generellen Verhältnismäßigkeitsanforderungen genügen. ${ }^{23}$ Diese abgestufte Auslegung trägt letztlich auch die zwei zentralen einschränkenden, von einigen der genannten Entscheidungskritiker indes als bloß vorgeschoben angesehenen Feststellungen des Bundesverfassungsgerichts: Sie stellt zum einen sicher, dass auch in diesem Ausnahmefall nicht eine bestimmte geistige Haltung allein zum Gegenstand einer Eingriffsmaßnahme gemacht werden kann und der Schutz der „Meinungsfreiheit als Geistesfreiheit" in seinem Kern unangetastet bleibt. Zum anderen findet in dieser Auslegung die Ablehnung eines generellen anti-nationalsozialistischen Grundprinzips eine Stütze; dementsprechend gilt die Rechtfertigung von Eingriffsakten, die sich gegen die Gutheißung der nationalsozialistischen Verbrechensherrschaft richten, nicht für die „zustimmende Bewertung von [anderen] Maßnahmen des nationalsozialistischen Regimes". 24

Die Argumentation des Bundesverfassungsgerichts steht allerdings vor dem Problem, dass sie eine historische Feststellung - der „Historikerstreit“ trägt seinen Namen aus gutem Grund - in einen juristischen (Ausnahme-)Tatbestand umformuliert. ${ }^{25}$ Diese Ar-

20 Zum Zusammenhang von Art. 5 Abs. 2 und Art. 3 GG sowie zur Geltung des Allgemeinheitserfordernisses auch für jugend- und ehrschützende Gesetze BVerfGE 124, 300 (324, 326 f., 338); pauschal zustimmend M. Hong, DVB1. 2010, 1267 (1270); undeutlich zum Verhältnis von Jugendschutz und Allgemeinheitserfordernis in Bezug auf § 184 StGB hingegen noch BVerfG, Beschl. v. 31.7.1989, Az. 1 BvR 1130/87 (unveröff.).

21 BVerfGE 124, 300 (331); kritisch zum diesbezüglichen Gefährdungspotenzial des von § 130 Abs. 4 StGB unter Strafe gestellten Verhaltens W. Höfling/S. Augsberg, JZ 2010, 1088 (1097); T. Holzner, DVBl. 2010, 48 (49f.); U. Volkmann, NJW 2010, 417 (420); vgl. dazu auch $A$. Scheidler, NWVBl. 2010, 131 (135); ders., apf 2010, 306 (311f.); zu einer gegenläufigen Folgeentscheidung M.W.H. Möllers, in: ders./R.C. van Ooyen (Hrsg.), Jahrbuch Öffentliche Sicherheit 2010/2011, 1. Halbbd., 2011, $173 \mathrm{ff}$.

22 BVerfGE 124, $300(326,329)$.

23 Dazu BVerfGE 124, 300 (331 ff.).

24 BVerfGE 124, 300 (330 f., 337, undifferenziert hingegen noch $327 \mathrm{f}$.); vgl. zum letztgenannten Fall - dementsprechend ohne strafrechtlichen Bezug - OLG Köln, AfP 2009, 603 ff.

25 BVerfGE 124, 300 (328 ff.), betont mehrfach die „historische“ Bedeutung der Ereignisse. 
gumentation ist nur dann tragfähig, wenn die Singularität der nationalsozialistischen Verbrechensherrschaft zugleich eine Verfassungsaussage darstellt. Das Bundesverfassungsgericht verweist dazu auf die Schöpfer des Grundgesetzes und ihr ,zentrales Anliegen“, einen bewussten „Gegenentwurf zu dem Totalitarismus des nationalsozialistischen Regimes“ zu schaffen. Das darin liegende ,innere Gerüst der grundgesetzlichen Ordnung“" komme besonders in Art. 1, 20 und 79 Abs. 3 GG zum Ausdruck. ${ }^{26}$ Überdies wird - allerdings möglicherweise unbewusst - mit der mehrfachen Bezugnahme auf die bleibende deutsche „Verantwortung“ für das nationalsozialistische Unrecht ${ }^{27}$ ein Begriff aus Satz 1 der GG-Präambel aufgegriffen. ${ }^{28}$ Zählt man die Absetzung vom nationalsozialistischen Unrechtsregime in dieser Weise zum Kern der „Gründungserzählung“29 grundgesetzlich verfasster Staatlichkeit, so wird man damit auch eine Heranziehung als historisch-teleologisches bzw. normativ angebundenes systematisches Argument bei der Auslegung von Einzelbestimmungen des Grundgesetzes begründen können. Nicht die historische Singularität des nationalsozialistischen Unrechts im Globalvergleich, um die es im „Historikerstreit“ ging, sondern sein besonderer Charakter als in singulärer Weise grundgesetzprägender Umstand bildet damit das maßgebliche Argument für das Bundesverfassungsgericht. Zumindest eine indirekte späte Stellungnahme im Historikerstreit formuliert das Bundesverfassungsgericht so gleichwohl. Mit seiner Anerkennung einer historisch begründeten Ausnahme vom Allgemeinheitserfordernis ermöglicht das Bundesverfassungsgericht eine spezifische Anknüpfung an die nationalsozialistische Verbrechensherrschaft in äußerungsbezogenen Rechtsnormen. Um dem absoluten Allgemeinheitsmaßstab zu genügen, wäre andernfalls nur eine generelle Bezugnahme auf „Verbrechensherrschaften“ möglich, im konkreten Fall also ein Verbot der Verherrlichung, Rechtfertigung oder Billigung totalitärer Herrschaften. Eine solche Fassung des $\S 130$ Abs. 4 StGB hätte auch ohne Anerkennung einer Ausnahme vom strengen Allgemeinheitserfordernis vor Art. 5 Abs. 2 GG Bestand gehabt, da davon nicht eine bestimmte politisch-inhaltliche Position - die Befürwortung einer irgendwie gearteten Verbrechensherrschaft reicht dafür nicht aus ${ }^{30}$ - erfasst wird. Wäre eine derart allgemein gefasste Formulierung geboten, so würde das im „Historikerstreit“ faktisch postulierte „Vergleichsverbot ${ }^{\star 31}$ nun rechtlich in sein genaues Gegenteil verkehrt. Ein derartiger rechtlicher Gleichsetzungszwang würde der „für die verfassungsmäßige Ordnung der Bundesrepublik Deutschland [...] gegenbildlich identitätsprägende[n] Bedeutung " 32 der nationalsozialistischen Verbrechensherrschaft nicht gerecht und wäre damit im Ergebnis genauso problematisch wie eine Verrechtlichung des früheren faktischen Postulats, zumal dieses im Prozess der „Historisierung des Historikerstreits“

26 BVerfGE 124, 300 (328); vgl. auch Fn. 32.

27 BVerfGE 124, $300(321,331,337)$.

28 Zur Ablehnung einer expliziten Bezugnahme der GG-Präambel auf die „Überwindung der NS-Herrschaft“ im Parlamentarischen Rat, um dem „Nationalsozialismus nicht an der Spitze des Grundgesetzes ein ewiges Denkmal [zu] setzen“, K. Naumann, Eine religiöse Referenz in einem Europäischen Verfassungsvertrag, 2008, S. 33 Fn. 15.

$29 \mathrm{Zu}$ einer der formalen Verfassungsgebung vorgelagerten Konstituierung der Staatlichkeit durch Gründungserzählungen vgl. (v.a. mit Bezug zu antiken Staatsgründungen) auch allgemeiner A. Koschorke, Neue Rundschau 1/2004, $40 \mathrm{ff}$.

30 C. Degenhart, JZ 2010, 306 (309).

31 E. Jesse, Cicero 10/2006, 62.

32 BVerfGE 124, 300 (328); vgl. auch Fn. 26. 
mittlerweile verbreitet relativiert wird. ${ }^{33}$ Die Auslegung des Art. 5 Abs. 2 GG durch das Bundesverfassungsgericht ermöglicht dem Gesetzgeber, der auch in rechtlicher Hinsicht unbestreitbar grundlegenden historischen Sonderkonstellation so Rechnung zu tragen, wie er es für richtig hält: Er kann sie durch allgemeines Gesetz - diese Möglichkeit muss unberührt bleiben - relativieren, ist dazu aber verfassungsrechtlich nicht gezwungen.

\section{Auswirkungen auf andere Fallkonstellationen}

Würde die Wunsiedel-Entscheidung das im Zuge des „Historikerstreits“ postulierte strikte Vergleichsverbot unbesehen übernehmen, so würde sich die Furcht vor einem damit geschaffenen Einfallstor für eine generelle Aufweichung des Allgemeinheitserfordernisses im Rahmen von Art. 5 Abs. 2 GG von selbst verbieten. Denn die Bezugnahme auf die historische Singularität würde dann eine Ausdehnung dieser Rechtsprechung auf andere Fallkonstellationen gerade nicht zulassen, sondern von vornherein ausschließen. Ganz so eindeutig ist die Entscheidung aber, wie gezeigt, nicht zu verstehen.

Das Bundesverfassungsgericht selbst hat sich zu den diesbezüglichen Entscheidungsfolgen zwar nur sehr knapp, aber im Ergebnis unmissverständlich geäußert, indem eine Übertragbarkeit ,auf andere Konflikte“ unter Verweis auf die Singularität ausdrücklich verneint wird. ${ }^{34}$ Besonders vor dem Hintergrund entsprechender Regelungen anderer Staaten, z.B. der Regelung des $§ 269 /$ B des ungarischen Strafgesetzbuchs (Tragen bestimmter nationalsozialistischer und kommunistischer Symbole), ${ }^{35}$ stellt sich gleichwohl die Frage, ob eine Ausdehnung der Strafbarkeit nach $\S 130$ Abs. 4 StGB auf die Gutheißung weiterer, konkret benannter Gewalt- und Willkürherrschaften zulässig wäre. An der fehlenden Allgemeinheit würde sich dadurch - sofern es sich nicht lediglich um beispielhafte Nennungen im Rahmen einer allgemein gefassten Regelung handelt nichts ändern, weil der „Sonderrechtscharakter einer standpunktbezogenen Regelung [...] nicht dadurch beseitigt werden [kann], dass weitere politische Standpunkte her-

33 Dahingehend zusammenfassend E. Jesse, Cicero 10/2006, 62 (64).

34 BVerfGE 124, 300 (329).

35 Zur EMRK-Widrigkeit dieser Bestimmung speziell im Hinblick auf den roten Stern EGMR, Urteil v. 8.7.2008, Nr. 33629/06 (Vajnai/Ungarn); dazu Á. Domahidi, EuR 2009, 410 ff.; M. Hong, ZaöRV 70 (2010), 73 (112 ff.). Eine unmittelbare Überprüfung der Wunsiedel-Entscheidung durch den EGMR - vgl. dazu auch S. Martini, JöR, n.F. 59 (2011), 279 (298 f.) kommt nach dem Tod des Beschwerdeführers - zur verfassungsprozessualen Relevanz BVerfGE 124, 300 (317 ff.) - nicht mehr in Betracht; zur Möglichkeit einer Verfahrensfortführung vor dem EGMR und der demgegenüber bislang allenfalls für die körperlichen Schutzrechte diskutierten Klagemöglichkeit von Rechtsnachfolgern im Todesfall vgl. (jew. m. Rspr.-Nachw.) D. Ehlers, in: ders. (Hrsg.), Europäische Grundrechte und Grundfreiheiten, 3. Aufl., 2009, § 2 Rn. 33; S. Kadelbach, in: D. Ehlers/F. Schoch (Hrsg.), Rechtsschutz im Öffentlichen Recht, 2009, § 5 Rn. 39, 45 f.; V. Röben, in: R. Grote/T. Marauhn (Hrsg.), EMRK/GG, 2006, Kap. 5 Rn. 12, 29. 
ausgegriffen und gegenüber anderen diskriminiert werden “ .36 Eine Rechtfertigung käme also allenfalls entsprechend der in der Wunsiedel-Entscheidung anerkannten Ausnahme vom Allgemeinheitserfordernis in Betracht.

In seiner Mauerschützen-Entscheidung hat das Bundesverfassungsgericht die „Tötung von ,Grenzverletzern" als extremes staatliches Unrecht" des kommunistischen DDR-Regimes qualifiziert und eine Übertragung der zur Aufarbeitung des nationalsozialistischen Unrechts entwickelten Ausnahme vom strikt formalen Verständnis des Rückwirkungsverbots gemäß Art. 103 Abs. 2 GG auf diesen Fall gebilligt. ${ }^{37}$ Dort ging es allerdings nicht um die Vergleichbarkeit von nationalsozialistischem und kommunistischem Regime insgesamt, sondern um konkret vergleichbare Unrechtssituationen. In Bezug auf die Frage einer entsprechenden Erweiterbarkeit des $\S 130$ Abs. 4 StGB kann die Anerkennung einer (weiteren) Ausnahme vom Allgemeinheitserfordernis nach den Kriterien der Wunsiedel-Entscheidung entgegen einem potenziellen ersten Eindruck nicht allein von dem historischen Systemvergleich abhängen, dessen Zulässigkeit Gegenstand des „Historikerstreits“ war. Die entscheidende Frage ist vielmehr, ob für das kommunistische Unrechtsregime ein in vergleichbarer Weise originär prägender Einfluss auf die grundgesetzliche Staatsordnung anzunehmen ist wie für die nationalsozialistische Verbrechensherrschaft. Dagegen spricht die Entstehungs- und Entwicklungsgeschichte des Grundgesetzes: Die DDR wurde erst nach dessen Schaffung gegründet. Zu einer Verfassungsneugebung gemäß Art. 146 GG, welche die DDR-Erfahrungen als insgesamt prägend hätte aufnehmen können, ist es im Zuge der Wiedervereinigung nicht gekommen. ${ }^{38}$ Der Beitritt nach Art. 23 GG a.F. hat das Grundgesetz im Wesentlichen unverändert gelassen.

Zwar hat der Prozess der Teilung und Wiedervereinigung für das Staatsverständnis der Bundesrepublik Deutschland durchaus ebenfalls eine prägende Bedeutung. Das zeigt schon die Präambel des ursprünglichen Gesetzes über den Tag der deutschen Einheit aus dem Jahre 1953, wonach der 17. Juni als Tag der Erhebung gegen die „,kommunistische Gewaltherrschaft [...] zum Symbol der deutschen Einheit in Freiheit geworden" ist. ${ }^{39}$ Diese symbolische Bedeutung wird in der Neufestlegung des Nationalfeiertags auf den 3. Oktober als „Tag der deutschen Einheit“ gemäß Art. 2 Abs. 2 des Einigungsvertrages von 1990 fortgeschrieben. Das ändert aber nichts daran, dass das Grundgesetz in seiner Gesamtheit nicht als spezifischer Gegenentwurf zum DDR-Regime zu verstehen ist und eine weitergehende rechtliche Aufladung dieser für die gegenwärtige Existenz Deutschlands ebenfalls in besonderer Weise prägenden Gegebenheit damit ausscheidet. Zumindest in ihrer rechtlichen Bedeutung unter dem Grundgesetz ist die nationalsozialistische Verbrechensherrschaft also tatsächlich singulär. Eine

36 M. Hong, ZaöRV 70 (2010), 73 (120); konkret in Bezug auf $\S 15$ Abs. 2 SächsVersG (in der vom VerfGH Sachsen, Urteil v. 19.4.2011, Az.: Vf. 74-II-10 [juris], aus formellen Gründen insgesamt für nichtig erklärten Neufassung), 13 Abs. 2, 3 VersammlG LSA ders., DVB1. 2010, 1267 (1274f.).

37 BVerfGE 95, 96 (130 ff., bes. 136). Eine Bezugnahme auf diese Rechtsprechung findet sich im vorliegenden Kontext ansatzweise auch bei M. Hong, ZaöRV 70 (2010), 73 (118, Fn. 152); L. Michael, ZJS 2010, 155 (164).

38 Näher dazu etwa H. Dreier, in: ders. (Hrsg.), Grundgesetz, Bd. III, 2. Aufl., 2008, Art. 146 Rn. $26 \mathrm{ff}$.

39 BGB1. I, S. 778. 
Neubelebung des „Historikerstreits“ ergibt sich aus dieser Feststellung des Bundesverfassungsgerichts aber trotz der insoweit missverständlichen Anklänge in der Begründung des Wunsiedel-Beschlusses nicht. 